

Carlos Javier Sánchez Zambrana*

\section{La integración en los Estudios Generales: reflexiones a partir una experiencia}

\author{
Reflections on the experience of integrating \\ knowledge into the field of General Studies
}

Recibido: 19-06-17 Aprobado: 29-08-17

\title{
Resumen
}

Este artículo comparte una experiencia curricular de particular significado en la Facultad de Estudios Generales de la Universidad de Puerto Rico. Se trata de la maduración y concreción del concepto curricular en torno a la integración del conocimiento en un seminario de cierre para un Programa que otorga grado en Educación General. Estamos convencidos que el arte vital para abordar adecuadamente e intentar solucionar las situaciones con las que nos encontramos en el mundo de hoy consiste en la integración, tanto en sus múltiples dimensiones (artística, científica, literaria, experiencial, emocional, espiritual) como en sus variadas fuentes de conocimiento (ancestrales, contemporáneas, sagradas y profanas). Sostenemos que son los Estudios Generales el componente curricular universitario que más ha aportado a esos procesos de interconexión entre saberes y entretejidos holísticos para propiciar la formación integral de los estudiantes. En este artículo nos proponemos discutir ciertas bases teóricas para el estudio de la integración del conocimiento dentro de las coordenadas de la educación general. Realizaremos, además, un recorrido histórico del tratamiento del tema y presentaremos algunos de los frutos de la experiencia aludida

\section{Abstract}

This article shares a curricular experience of particular significance in the Faculty of General Studies at the University of Puerto Rico. It discusses the development and concretion of the curricular concept around the integration of knowledge in a closing seminar for a program that leads to a degree in General Education. We are convinced that the solutions to most situations we come across in today's world require integrating multiple dimensions (artistic, scientific, literary, experiential, emotional, spiritual) and varied sources of knowledge (ancestral, contemporary, sacred and profane). We posit General Studies is the university curriculum component that has contributed the most to processes that require weaving together different types of knowledge for the purposes of a holistic training. The article discuss several theoretical bases for the study of the integration of knowledge into the General Education degree. Furthermore, it analyzes the historical evolution of the topic and presents findings on the results of the integration experience.

Palabras clave

Educación General; Integración; Transdisciplinariedad; Seminarios; Estudios Generales; Currículo

\section{Keyword}

General education; integration; transdisciplinary; Seminars; general studies; Curriculum

*Carlos Javier Sánchez Zambrana: Doctorado en la Universidad Nacional Autónoma de México. Es catedrático en la Facultad de Estudios Generales en la Universidad de Puerto Rico. Para contactar al autor: carlos.sanchez8@upr.edu 


\section{Introducción}

En este artículo realizaremos un breve recorrido histórico del tratamiento del tema sobre la integración del conocimiento en la Educación General y presentaremos los frutos de una experiencia concreta en la Facultad de Estudios Generales (FEG) de la Universidad de Puerto Rico (UPR).

Espero que sorprenda al lector tanto como al autor, la falta de correspondencia entre el escaso cultivo del tema en contraste con su crucialidad para los Estudios Generales.

En tanto la Facultad de Estudios Generales (FEG) produjo una institucionalidad y constituyó una comunidad organizada, desde 1945, la consigna en pro de integración, se estableció como un elemento constante en nuestra cotidianidad y en nuestros discursos. Se escuchará hablar pues, de integración en su máxima amplitud y laxitud a la vez: a) como sinónimo de articulación en cuanto a los componentes de educación general y las especialidades, curricularmente hablando; b) como sucedáneo de "socialización" al dirigirse la integración hacia asuntos de enfoques y experiencias del cuerpo docente, de los servicios al estudiante, etc. y c) en el sentido epistémico como tejido de materias y saberes, del conocimiento entre sí y contra sí. Este último plano, aunque cultivado menos de lo deseado, es el terreno predilecto -natural pero contingente- para la evolución de la educación general.

El problema fundamental que surge al pensar en contenidos curriculares concretos y en concepciones filosóficas adviene de la inquietud sobre la ontogénesis de la integración del conocimiento en la educación general. Este concepto, como eje o nudo de tensión problemática en la historia de la Educación General en Puerto Rico está vinculado a por lo menos dos complexus:

a) con la idea de que existe interdependencia entre los elementos constitutivos en una relación educacional: por un lado, el sujeto cognoscente en su personal idiosincrasia y situación existencial, por otro lado, el conocimiento como un corpus externo organizado en un currículo que proviene de instituciones, de la cultura y de la tradición histórica. Si nos asomamos al mundo interior del sujeto cognoscente ¿cómo es que conoce la realidad, en qué consiste "conocer" los contenidos de lo real en su conciencia? La pregunta, que aún no tiene respuestas determinantes en filosofía ni en ciencias cognitivas, permea ciertamente todo el drama educativo. b) Y con la idea de la integración amplia y abierta en el sentido en que Pascal -como pionero en esta concepción epistemológica- concibió las interrelaciones entre el todo y sus partes:

...siendo las cosas causadas y causantes, ayudadas y ayudantes, mediatas e inmediatas, y todas entretejiéndose por un lazo natural e insensible que liga las más lejanas y las más diferentes, yo considero imposible conocer las partes sin conocer el todo, tanto como conocer el todo sin conocer particularmente las partes. (Pascal, [1656] 1940).

¿Cómo se integran "conocimientos" de educación general, en el sujeto que aprende y los sujetos que educan? Para integrar es necesario vincular al educando-educador en relaciones que hagan posible la "educación en educación general", lo que implica, como mínimo, establecer bases de solidaridadconfianzas interpersonales- de un proceso educativo abierto, colaborativo y experimental; identificar nexos epistémicos entre los saberes que estudian dimensiones de lo real; orquestar una pedagogía holista que articule didáctica/aprendizaje como proceso unitario y recíproco; y que los sujetos educandos puedan incorporar nuevos saberes a los que poseían anteriormente, reestructurando su universo interior y aplicando los saberes integrados a nuevas situaciones concretas de su cotidianidad.

Vélez Cardona -uno de los principales cultivadores del estudio de las redes entre integración, transdisciplinariedad y educación general- intenta esclarecer el camino. Señala:

En la segunda mitad del siglo XX se insistió en que una integración efectiva de elementos cognitivamente heterogéneos en un nuevo paradigma epistémico estaba estrechamente vinculada con la transformación de la comunidad científica. Eso explica el auge de la interdisciplinariedad primero y de la transdisciplinariedad después, como un importante desarrollo histórico en el ámbito de la integración del conocimiento. Particularmente, en momentos en los que las disciplinas están experimentando tremendos cambios, los que se caracterizan por prácticas más pluralistas, el cruce de fronteras, su apertura a la interdisciplinariedad y su atención a la complejidad para poder resolver los problemas actuales (Vélez Cardona, 2011, pp. 5-6).

Es por eso que el primer paso en la integración es reconocer y respetar la diversidad de perspectivas. Una 
de las principales voces pioneras en trabar los términos de la conversación para alertar a favor de los nexos y conexiones entre lo interdisciplinario y la integración del conocimiento ha sido Julie Thomson Klein. La diversidad no se ha de considerar ahora -nos señalacomo una limitación, sino más bien, como una invitación para la interacción creativa y productiva (Klein, 2011, p. 414). Esta comprensión de la vida engrana con actitudes de humildad científica y rememora la voz de Joan Manuel Serrat al reconocer el valor de "aquellas pequeñas cosas", mismas que reflejan la situación del ser humano en el mundo: "minúscula parte del todo pero que lleva la presencia del todo en esta minúscula parte" (Morin, 1999, 2002, p. 43).

\section{Ojeada al proceso histórico}

A fines de los años sesenta y con una mirada retrospectiva del "fulgor" del movimiento intelectual de la década anterior, el importante sociólogo Daniel Bell formulaba sobre las venturas y desventuras de la integración en la Educación General:

Lo que estamos contemplando en la actualidad es que se echa a un lado la Educación General y se reemplaza por un sistema de cafetería en el que cualquier clase de curso es admitido como parte del corpus de la Educación General, hasta el nivel que los estudiantes toman algún (si alguno) curso fuera de su especialización. En el lugar de la Educación General tenemos "requisitos distribucionales" en el que los estudiantes tienen que tomar cualquier curso de ciencia, cualquier curso de humanidades, cualquier curso de ciencias sociales, con poca preocupación por la lógica, coherencia y relevancia intelectual o cualquier otro criterio a excepción de estas tres etiquetas. Esta desintegración del curriculum se debe, en parte, a razones intelectuales. Las esperanzas de la integración interdisciplinaria, muy grande 20 años atrás, no se ha materializado, por lo menos al nivel de la Educación General (Bell, 1968 c.1966 p.23).

En el contexto norteamericano en el cual inexorablemente gravitó la Universidad de Puerto Rico, y que tan expedita entrada tenía y tiene en nuestros lares, el tema de la integración fue cultivado por el canon del movimiento de Educación General. En 1945 sale a la luz pública el informe de la Universidad de Harvard, General Education in a Free Society [conocido como el Red book], en el mismo se establece como criterio fundamental para la educación general la integración del pensamiento y la acción dentro de un marco que denominaban el hombre libre en una sociedad libre. (Harvard Committee, 1946).

Por los mismos años, Earl James McGraf, importante editor del Journal of General Education, quien luego se convertiría en Secretario de Educación de los EEUU para dos presidentes y quien visitará la FEG a mediados de la siguiente década, hacía la admonición siguiente:

La reacción contra el especialismo y el vocacionalismo es acompañada de un esfuerzo por integrar las materias de disciplinas relacionadas. Los bordes entre departamentos estrechamente relacionados son guardados más cuidadosamente que las fronteras de naciones hostiles (McGrath, 1946, pp. 3-8).

Nos parece adecuado asentar por nuestra parte que los extremos de esta polémica en los comienzos de la década de los cincuenta establecían contornos entre el especialismo y cierta visión de educación general que buscaba superar las limitaciones del primero haciendo hincapié en aspectos de nexos y correlaciones entre los saberes. Parecía un asunto de escogencia ideológica: por un lado, la visión de tipo hipermercado que privilegia las necesidades por un nicho o espacio específico, y especial en y para las ciencias, y por el otro, las búsquedas por coherencia e integración curricular en y para el estudiante, en correlato polar al interior de la misma ecuación.

Mientras, en la FEG se buscaba la piedra identitaria; la puja por la ontogénesis conducía a explorar con la integración en múltiples sentidos y más allá de las estructuras. Tan es así, que es posible afirmar que durante el primer lustro de la década de los cincuenta la actividad de mayor énfasis, a la que se le dio atención especial en las reuniones de Facultad y en los seminarios fue el problema de la integración de los cursos básicos.

En efecto, durante los primeros años de la década, el asunto de la integración fue adquiriendo centralidad en varios frentes: a) en los foros de literatura teórica en el campo; b) en el espacio deliberativo de la Junta Universitaria del Recinto a tenor con la revisión de los programas de estudios que presentaban los Decanos de Facultades y c) en ciertas instancias -Asambleas, Comités y Seminarios- que se iban aperturando en la FEG. Como cuestión de hecho, el decano de la FEG para la década de los cincuenta, Ángel Quintero Alfaro, atizó constantemente el tema de la integración. Solía establecer por lo menos cuatro enfoques para el desarrollo de la integración: a) la integración por asimilación; b) integración por adhesión; c) integración individual; $y \quad d)$ integración por comprensión de diferencias y semejanzas (Informe Anual de la Facultad de Estudios Generales, 1954-55).

En una línea de investigación activa abordamos estas dimensiones con cierto detalle y proveemos las fuentes específicas para su estudio. Nos referimos a la serie de entregas bajo el concepto de Historia Crítica de la Educación General en Puerto Rico (Maldonado, Vélez \& Sánchez, 2013). Ha sido publicado el volumen referente a los antecedentes y la etapa fundacional. 
Está en turno el tomo dedicado precisamente a esta crucial década de los cincuenta del siglo pasado.

A finales de los años ochenta y principios de noventa se percibió la necesidad de revisar -una vez más-1 las metas y objetivos en la FEG. Tal vez lo más terrible era -expresan voces de la época- que dentro de la propia Facultad se había perdido el sentido, el entendimiento y el convencimiento de las virtudes y las limitaciones de la educación general ${ }^{2}$. En ese contexto, la FEG se propone realizar un auto-estudio, que comienza en 1989 y en el cual toma auge el tema de la interdisciplinariedad. Aquí se plantea que la identidad de la FEG no le viene de la interdisciplinariedad, sino de la integración del conocimiento. La interdisciplinariedad se entendería como un método, como un instrumento para lograr la integración del conocimiento.

Estas aporías representan apenas la punta de un témpano en las discusiones permanentes y críticas que los Estudios Generales deben promover para revisitar constantemente sus supuestos y alcances. Así las cosas y a riesgo de realizar saltos cronológicos de malabar, quisiera compartir el eslabón más reciente de esta cadena que, como vimos, hunde sus raíces en las décadas fundacionales de la FEG. Se trata de la maduración y concreción del concepto curricular en torno a la integración del conocimiento: un curso tipo seminario de cierre, requisito para los estudiantes que concluyen su grado de Bachiller en Artes (licenciatura) en Estudios Generales. Vale acotar que el término bachillerato y el grado de Bachiller se emplean según la semiótica imperante en la UPR derivada del contexto norteamericano. Para América Latina debe entenderse como equivalente al grado de licenciatura.

\section{La integración del conocimiento como Seminario o Programa de Estudios}

Bajo el título de Seminario de Integración Interdisciplinaria del Conocimiento (ESGE4305) y como producto de una revisión curricular, se comienza -en 2015-16- a ofrecer una experiencia docente capstone, para los estudiantes que cursan su cuarto año curricular en el Programa de Bachillerato en Artes de la Facultad de Estudios Generales. El Seminario se desarrollará a partir de la integración del conocimiento, en su modalidad interdisciplinaria y considerando su evolución hasta llegar a lo transdisciplinar. En la medida en que sostenemos que el principal fundamento de la Educación General es la integración del conocimiento, el Seminario abordará las limitaciones de los esquemas multidisciplinarios. Es este norte curricular -la integración- el que permite avanzar hacia la vinculación interdisciplinaria de las diversas áreas del saber. Los objetivos del curso, que muy bien podrían ser también los de un Seminario de formación docente permanente, se explicitan con el siguiente orden de ideas ${ }^{3}$ :

A. Se reflexionará sobre las limitaciones de las disciplinas, vistas de manera aislada y pretendidamente autosuficientes, así como de la importancia de superar dichas limitaciones para abordar y solucionar los problemas más apremiantes que enfrentamos en el Siglo XXI.

B. Se desarrollarán experiencias educativas conducentes a la conceptuación de la integración del conocimiento interdisciplinario y sus aplicaciones prácticas tanto en el mundo académico como en la vida cotidiana.

C. Se iniciará la marcha hacia el desarrollo de altos niveles de comprensión en torno a los procesos de integración del conocimiento inter y transdisciplinarios, su importancia, sus obstáculos, metodologías y tendencias contemporáneas.

D. Se fomentará el interés por la investigación inter y transdisciplinaria en las que se integran y hasta trascienden las diversas disciplinas y áreas del saber. En ese afán, se trabajarán destrezas de investigación que tomen en consideración y comiencen a integrar las diversas dimensiones de los asuntos o problemas que despierten su interés, particularmente en el mundo académico, pero intentando aplicarlos a las demás dimensiones de su vida.

El temario del Seminario discurre en armonía con los objetivos. Así pues, podrían desplegarse cuatro unidades centrales, a saber:

A. La integración del conocimiento.

1. ¿Qué debemos entender por integración del conocimiento? ¿Por qué no una definición?

2. El devenir histórico de este concepto.

3. Las diversas maneras en que se ha procurado desarrollarlas.

4. Su relación histórica con la Educación General.

B. La interdisciplinaridad para la integración del conocimiento.

1. Particularidades y limitaciones de la interdisciplinariedad.

2. Sus fundamentos epistemológicos.

\footnotetext{
${ }^{1}$ Hubo auto-evaluaciones anteriores como el denominado Proceso de Reevaluación de la Facultad de Estudios Generales de 1973.

${ }^{2}$ Entrevista semi-Estructurada realizada a la profesora y ex Decana jubilada de la FEG, Dra. Josefina Iglesias (mayo 2012).

${ }^{3}$ Para ver las especificidades de este curso en su formato de Prontuario acceda a la página electrónica de la FEG: generales.uprrp.edu/ Bachillerato.
} 
3. Auge y decadencia de la interdisciplinariedad.

4. Su relación con la Educación General.

C. La transdisciplinariedad para la integración del conocimiento.

1. Particularidades y limitaciones de la transdisciplinariedad.

2. Sus fundamentos epistemológicos.

3. Origen y desarrollo de la transdisciplinariedad.

4. Su relación con la Educación General.

D. Aplicaciones prácticas de la integración del conocimiento

1. La investigación interdisciplinaria en la academia.

2. La investigación transdisciplinaria y el conocimiento socialmente robusto.

3. Particularidades de la investigación en los Estudios Generales (Tesis, ensayos etc.).

4. Cultivo práctico de las destrezas necesarias para producir investigaciones que integren conocimientos inter y transdisciplinariamente (talleres).

\section{Conclusiones:}

A modo de cierre preliminar, quisiéramos dejar asentado que esta experiencia constituye una de las múltiples maneras de emprender curricularmente el tema de la integración del conocimiento desde la perspectiva de la Educación General transdisciplinaria. Esta propuesta es, en términos específicos, fruto de un trabajo colaborativo que en los años recientes el Dr. Waldemiro Vélez Cardona y este servidor hemos realizado en los Seminarios de Educación General de la FEG y el Departamento de Ciencias Sociales. Empero, en sentido amplio, es parte de una historia zigzagueante en donde la integración como aporía y norte, no siempre ha sido valorada con las mismas energías. Hubo etapas en donde pareció existir consciencia de su crucialidad, y otras en donde abandonaba la palestra, más allá de lo aconsejable para una Facultad de Estudios Generales.

Sin embargo, y de modo optimista, pienso que estamos en un gran momento para retomar su pertinencia: la policrisis. Urgidos de producir una crítica superadora y constructiva para el robustecimiento académico de nuestros estudiantes, de todo ciudadano solidario y de nuestra sociedad, nada más pertinente que re-convocarnos al examen de la integración en sus múltiples valencias.

¿Qué resultados se han obtenido a partir de la reforma del Seminario de Integración? ¿Cómo ha repercutido en la Institución y en la formación del estudiante del Programa de Bachillerato (Licenciatura) en Educación General? ¿Qué nos muestra el necesario proceso de avalúo? ¿Qué recomendaciones se extraen para lograr de este proceso curricular uno de reforma permanente? Estas preguntas, ciertamente, quedan como reto y como carta de navegación para el siguiente escorzo.

\section{Referencias}

Bell, D. (1968 c.1966). The Reforming of General Education. New York: Anchor Books, Doubleday \& Company, Inc.

Facultad de Estudios Generales. (1955) 1). Informe Anual 1954-1955. Río Piedras: Universidad de Puerto Rico

Harvard Committee. (1946). General Education in a free Society: Report of the Harvard Committee. Harvard University Press.

Iglesias, J. (1997, 8 de octubre). La integración del conocimiento en la educación general. En Seminario sobre Educación General. San Juan, PR: Universidad de Puerto Rico.

Klein, J. T. (2011). Research Integration. A comparative knowledge Base. En A. F. Repko; W. H. Newell, y R. Szostak (Eds.) Case studies in Interdisciplinary Research. London: Sage.

Maldonado, N., Vélez, W., y Sánchez, C. (2013). Historia crítica de la Educación General en Puerto Rico. San Juan, PR: Ediciones Mágica.

McGrath, J. E. (1946). The General Education Movement (An Editorial). The Journal of General Education, 1(1), 3-8.

Morin, E. (1999, 2002). La cabeza bien puesta: repensar la reforma; reformar el pensamiento. Buenos Aires: Nueva Visión.

Pascal, B. (1940/1656). Pensamientos. Madrid: Espasa Calpe.

Universidad de Puerto Rico (2013-014) Prontuario del curso ESGE 4305. (2013-014) Facultad de Estudios Generales. San Juan

Vélez Cardona, W. (2013). La integración del conocimiento como fundamento de los estudios generales. En Documentos 21. Santo Domingo: Instituto Tecnológico de Santo Domingo.

Vélez Cardona, W. (2012). ¿Educación general o estudios interdisciplinarios? En IV Simposio Internacional de Estudios Generales. Lima, Perú.

Vélez Cardona, W. (2011). Una educación general transdisciplinaria para el fortalecimiento de la universidad. UMBRAL, (6), 5-32. 